\title{
Hockey Concussion Education Project, Part 1. Susceptibility-weighted imaging study in male and female ice hockey players over a single season
}

\author{
Clinical article
}

\author{
Karl G. Helmer, Ph.D., ${ }^{1}$ Ofer Pasternak, Ph.D., ${ }^{2}$ Eli Fredman, B.A., ${ }^{2}$ \\ Ronny I. Preciado, B.A., ${ }^{13}$ Inga K. Koerte, M.D., ${ }^{2,3}$ TAKeshi SaSaki, M.D., Ph.D., ${ }^{2,4}$ \\ Michael Mayinger, ${ }^{2,3}$ Andrew M. Johnson, Ph.D., ${ }^{5}$ Jeffrey D. Holmes, Ph.D., ${ }^{6}$ \\ Lorie A. Forwell, M.P.T. ${ }^{7,8}$ Elaine N. Skopelja, M.A.L.S., 9 *MARTha E. Shenton, Ph.D., ${ }^{2,10,11}$ \\ and Paul S. Echlin, M.D.12
}

${ }^{1}$ Massachusetts General Hospital/Massachusetts Institute of Technology/Harvard Medical School/ ${ }^{13}$ Athinoula A. Martinos Center for Biomedical Imaging, Charlestown, Massachusetts; ${ }^{2}$ Psychiatry Neuroimaging Laboratory, Department of Psychiatry, Brigham and Women's Hospital/Harvard Medical School, Boston, Massachusetts; ${ }^{3}$ Institute for Clinical Radiology, Ludwig-Maximilians-University, Munich, Germany; ${ }^{4}$ Department of Psychiatry and Behavioral Sciences, Tokyo Medical and Dental University Graduate School, Tokyo, Japan; ${ }^{5}$ School of Health Studies, ${ }^{6}$ School of Occupational Therapy, ${ }^{7}$ Fowler Kennedy Sport Medicine Clinic, and ${ }^{8}$ School of Physical Therapy, The University of Western Ontario, London, Ontario; ${ }^{9}$ Ruth Lilly Medical Library, Indiana University, Indianapolis, Indiana; ${ }^{10}$ VA Boston Healthcare System, Brockton, Massachusetts; ${ }^{11}$ Department of Radiology, Brigham and Women's Hospital/Harvard Medical School, Boston, Massachusetts; and ${ }^{12}$ The Elliott Sports Medicine Clinic, Burlington, Ontario, Canada

Object. Concussion, or mild traumatic brain injury (mTBI), is a commonly occurring sports-related injury, especially in contact sports such as hockey. Cerebral microbleeds (CMBs), which appear as small, hypointense lesions on $\mathrm{T}_{2}{ }^{*}$-weighted images, can result from TBI. The authors use susceptibility-weighted imaging (SWI) to automatically detect small hypointensities that may be subtle signs of chronic and acute damage due to both subconcussive and concussive injury. The goal was to investigate how the burden of these hypointensities changes over time, over a playing season, and postconcussion, in comparison with subjects who did not suffer a medically observed and diagnosed concussion.

Methods. Images were obtained in 45 university-level adult male and female ice hockey players before and after a single Canadian Interuniversity Sports season. In addition, 11 subjects ( 5 men and 6 women) underwent imaging at 72 hours, 2 weeks, and 2 months after concussion. To identify subtle changes in brain tissue and potential CMBs, nonvessel clusters of hypointensities on SWI were automatically identified, and a hypointensity burden index was calculated for all subjects at the beginning of the season (BOS), the end of the season (EOS), and at postconcussion time points (where applicable).

Results. A statistically significant increase in the hypointensity burden, relative to the BOS, was observed for male subjects with concussions at the 2-week postconcussion time point. A smaller, nonsignificant rise in the burden for female subjects with concussions was also observed within the same time period. There were no significant changes in burden for nonconcussed subjects of either sex between the BOS and EOS time points. However, there was a statistically significant difference in the burden between male and female subjects in the nonconcussed group at both the BOS and EOS time points, with males having a higher burden.

Conclusions. This method extends the utility of SWI from the enhancement and detection of larger (> $5 \mathrm{~mm}) \mathrm{CMBs}$, which are often observed in more severe cases of TBI, to cases involving smaller lesions in which visual detection of injury is difficult. The hypointensity burden metric proposed here shows statistically significant changes over time in the male subjects. A smaller, nonsignificant increase in the burden metric was observed in the female subjects. (http://thejns.org/doi/abs/10.3171/2013.12.JNS132093)

\section{KeY Words • susceptibility-weighted imaging • ice hockey $\bullet \quad$ sex-based difference $\bullet$ concussion - mild traumatic brain injury}

\footnotetext{
Abbreviations used in this paper: $\mathrm{BOS}=$ beginning of the season; $\mathrm{CMB}=$ cerebral microbleed; $\mathrm{DWI}=$ diffusion-weighted imaging; $\mathrm{EOS}=$ end of the season; GRE = gradient recalled echo; $\mathrm{HIB}=$ hypointensity burden; ImPACT = Immediate Postconcussion Assessment and Cognitive Test; $\mathrm{mTBI}=$ mild traumatic brain injury; SCAT2 = Sport Concussion Assessment Tool-2; SWI = susceptibility-weighted imaging.

* Drs. Shenton and Echlin share senior authorship of this work.
}

I $\mathrm{T}$ is estimated that there are 1.7 million traumatic brain injury (TBI) occurrences from all causes per year and that emergency department visits and hospitalizations due to TBI are increasing..$^{18}$ This number does not include instances of TBI that are either treated by a primary care physician or are left untreated, so the actual number is expected to be higher. Sports are increasingly recognized as a significant source of TBIs in the young adult popu- 
lation. ${ }^{11}$ These sports-related TBIs are predominantly in the form of a concussion, also called a mild TBI (mTBI). Concussions may or may not involve loss of consciousness, and symptoms may be physical, somatic, sleep related, emotional, and/or cognitive. ${ }^{11,35}$ The sequelae from concussion are difficult to measure and the results from noninvasive imaging modalities such as CT scanning or conventional MRI may be normal for most patients. ${ }^{6}$ In addition, many sports such as soccer and hockey provide ample opportunity for the player to experience repeated subconcussive blows to the head, and the long-term effects may be related to cumulative damage. ${ }^{12,34}$

Damage due to concussions may arise both from the primary phase (the actual impact) and a secondary phase (postimpact). The damage at impact can be understood by using models of physics in which kinetic energy is transferred between players or a player and an object. In an analysis of game films from the US National Football League, forces were extracted and modeled. It was found that the highest strain forces were present at the deep midbrain level..$^{42,43}$ These forces are believed to also produce axonal injury in concussion, ${ }^{32,48}$ such as is more commonly observed in more severe TBI. ${ }^{46}$ In addition, cerebral microbleeds $(\mathrm{CMBs})^{40}$ have been observed to accompany axonal shearing, ${ }^{37}$ and both have been found to be nonlocalized. The secondary injury phase may result in the brain entering into a hypometabolic state, brain hypoperfusion, and a disruption of the blood-brain barrier resulting in brain edema. ${ }^{12}$

It has been observed that each sex responds to concussion differently and that the absolute rates of concussion have been shown to be greater for women than men in certain sports..$^{14}$ In hockey, for example, female players have a higher concussion rate than do male players. This is the case even given that intentional body checking is not allowed for females. ${ }^{1,2,16,45}$ Although the cause is not known, it has also been shown that the outcome for TBI is worse for females than for males. ${ }^{17}$ Complicating the interpretation of sex-based TBI differences and recovery are studies that have shown differences in neuropsychological baseline measures..$^{5,9}$

There are several different approaches that one can take to detect pathology in tissue. First, structural imaging can be used to take high-resolution images of the brain with $\mathrm{T}_{1^{-}}$and $\mathrm{T}_{2}$-weighted contrast enhancement. These data can then be used to extract the shape of cortical and subcortical regions, and differences can be calculated between these shapes and those of an atlas made from the brains of healthy individuals. Alternatively, one can search for intensity differences in these weighted images that indicate pathology. ${ }^{20,21}$ Another form of structural imaging that focuses on tissue microstructure is diffusionweighted imaging (DWI). In DWI, the microstructure of the tissue is interrogated using the diffusive motion of sensitized, endogenous water molecules. By measuring the net motion of the tissue water one can infer the integrity of the structures. Given this, DWI has been used to search for evidence of diffuse axonal injury ${ }^{37}$ in concussion. ${ }^{31,35}$ Other standard imaging methods include FLAIR imaging, which has been shown to be sensitive for the detection of cortical contusions and subdural hematomas, ${ }^{3}$ and $\mathrm{T}_{2}{ }^{*}$-weighted gradient recalled echo (GRE) scans, which are sensitive to hemorrhagic regions ${ }^{7,28}$ due to the paramagnetic nature of blood breakdown products, for example hemosiderin. However, standard MRI methods have shown mixed results when investigators have tried to correlate imaging results with clinical outcomes. ${ }^{15,25,26,33,49}$

In addition to structural measurements, one can use MRI to detect CMBs that often accompany TBI over a range of severity. Susceptibility-weighted imaging (SWI) is a relatively new MRI modality that involves further processing of a GRE data set. ${ }^{22-24,38}$ The contrast found in SWI is generated through the sensitivity of the phase measurement to disruptions in the magnetic field. ${ }^{23}$ The phase is sensitive to differences in magnetic susceptibility that, in the case of TBI, are provided by the iron present in pools of static blood resulting from (micro)hemorrhages. The SWI modality takes these phase disruptions and uses them to generate contrast that can greatly enhance the detection of small pockets of blood. ${ }^{8}$ The sensitivity of SWI is greater than that of a standard GRE sequence in which the contrast is related to the $\mathrm{T} 2 *$ distribution in the tissue..$^{39}$ The current study is the first to use SWI to assess the effects of sports concussion in a prospective manner.

In the case of concussion, it is not expected that there will be the type of large CMBs $(>5.7 \mathrm{~mm}$ has been suggested for visual inspection studies ${ }^{39}$ ) that have been observed to accompany stroke or severe TBI. Instead, we use SWI to detect smaller, more subtle intensity changes in the sensitized images. Our method, described below, is to interrogate the time course of clusters of low-intensity voxels. Through this method we can detect changes in the number of low-intensity clusters that may occur in response to concussion, and we can also investigate the initial burden of these clusters in each sex, how the clusters correlate with numbers of previous concussions, and how they change over time when no further concussion is reported.

\section{Methods}

\section{Study Subjects}

A cohort of 45 adult hockey players (25 men and 20 women) underwent imaging at the beginning of the season (BOS), the end of the season (EOS), and at 72 hours, 2 weeks, and 2 months postconcussion using an SWI protocol. Data from these subjects were processed and analyzed. However, not all of the participants who suffered a concussion during the season underwent imaging at each time point, due to subject withdrawal from the study or scheduling conflicts. For example, 4 of the male subjects were not included in the study either due to claustrophobia at imaging, self-withdrawal from the study, or noncompletion of the BOS imaging. The mean age of the participants at the BOS was $23 \pm 2$ years for the men and $21 \pm 4$ years for the women. From these 2 groups there were 5 confirmed concussions for the men and 6 for the women during the season. The internal review boards of the participating universities approved this study.

Previous concussions were self-reported by these subjects. Of the 16 men who did not have concussions during the season, 3 had 2 previous concussions and 5 had 1 previous concussion. Of the 14 women who did not have 
concussions during the season, only 1 had had a previous concussion. Several of the subjects who suffered a concussion during the season of interest also had previous concussions. Of the 5 men who had at least 1 concussion during the season ( 1 of the injured men had 2 concussions during the season between the 2-week and 2-month time points, and hence did not complete the 2-month and EOS time points), 2 of them had 1 previous concussion, 2 of them had 2 concussions, and a single subject reported 3 previous concussions. Of the 6 women who had a concussion during the season, 3 of them had no previous concussions, 1 had 1 previous concussion, 1 had 3 previous concussions, and a single subject reported 4 previous concussions.

\section{Imaging Protocol}

Subjects underwent imaging on a 3-T Philips Achieva equipped with an 8-channel head coil. The SWI data set was acquired using a multiecho gradient-echo pulse sequence consisting of 5 echoes, with an initial TE of 6 msec; the interval between echoes was $6 \mathrm{msec}$. To generate the SWI volumes analyzed here, the TE $30 \mathrm{msec}$ data were used. The TR was $34 \mathrm{msec}$. Slices were acquired in the transverse plane and the acquired FOV was $220 \times 167$ $\times 128 \mathrm{~mm}$ with a voxel volume of $0.5 \times 0.5 \times 2 \mathrm{~mm}^{3}$. This was then reconstructed to a voxel volume of $0.491 \times 0.491$ $\times 1 \mathrm{~mm}^{3}$. A SENSE factor of 1.2 was used in the rightleft direction, and the partial Fourier k-space acquisition percentage factor was $67 \%$. The flip angle was $17^{\circ}$ and the total acquisition time was 6 minutes and 39 seconds. The magnitude and phase images were saved for each volume in the PAR/REC format of the Philips system and were used in the SWI processing. It is also to be noted that a gradient coil change occurred during the collection of these data. Its effect on the imaging data and the final SWI volume voxel intensities was investigated (see below). However, because our goal here was to detect nonspecific "hypointensity" rather than to compare calculated parameter values (such as the apparent diffusion coefficient or the spin-spin relaxation time), it was not expected that the coil change would significantly affect these results. Nonetheless, analysis of the image intensity range and statistics was undertaken to ensure that this was in fact the case.

\section{Image Processing}

The SWIs were processed using in-house code written in $\mathrm{C}++$ and incorporated functions from the FreeSurfer libraries..$^{10}$ First, background pixels were removed from the magnitude volumes using the Brain Extraction Tool (BET) from the Functional MRI of the Brain (FMRIB) Software Library (FSL) ${ }^{47} \mathrm{~A}$ mask was then generated from the resulting brain-only volume and applied to the phase volume before the phase was unwrapped using the PRELUDE method, also from Functional MRI of the Brain (FMRIB) Software Library (FSL). ${ }^{29}$ Seven initial phase splits were chosen as an input to PRELUDE, and that value was selected as a compromise between speed of processing and quality of the resulting phase unwrapping. To create the SWI volume, the following steps were performed: 1) a gaussian smoothing filter of 2-mm kernel width was applied to the unwrapped phase volume; 2) the resulting smoothed volume was subtracted from the original phase volume, resulting in an effectively high-pass filtered phase volume; 3) a negative phase mask of the form for $\varphi>0 \rightarrow$ $\varphi=1$; for $\varphi \leq 0 \rightarrow \varphi=1+(\varphi / \pi)$, where $\varphi$ is the phase, was multiplied by itself 4 times and applied to the high-pass filtered unwrapped phase mask; and 4) this result was then multiplied by the masked magnitude volume, resulting in the final SWI volume. In addition, a minimum-intensity projection over 4 slices in the imaging-volume slice direction was performed and used to identify vessels, and hence the maximum-intensity threshold described in the following section.

\section{Image Analysis}

Analysis of the SWI volumes was performed using in-house code written in Matlab (The Mathworks, Inc.). Our hypothesis was that there would be few or no large hypointense regions that could be identified through visual inspection, and therefore our analysis focused on detecting smaller hypointensities that would be difficult to detect through visual inspection. In this analysis, the intensity that corresponded to maximum value of the lowest $10 \%$ of pixel intensities was used as a threshold value, and each voxel whose intensity value was at or below that level was recorded. In addition, the code also calculated the minimum/maximum/mean/median of the SWI voxel intensities for each subject to determine whether different image intensity ranges existed in the data for different cohorts or across the coil change date.

The algorithm then identified connected clusters of these marked voxels by using the 26-neighbor criterion for 3D space. This not only includes the closest neighboring voxels, but also neighboring voxels on the diagonal. To reduce the possibility of any vessels being included in these clusters, an upper limit on the cluster size included in the analysis was set at 35 voxels. The exact number chosen for either the cluster-size threshold or the imageintensity threshold was not found to be critical to the results presented here. Various cluster and intensity thresholds were chosen and similar results were found for all (data not shown), as long as the cluster threshold was low enough not to include obvious vessels that were identified as such by following their path manually. In addition, this cluster threshold corresponds roughly to a 3-voxel $\times 4$-voxel hypointense region that is connected across 3 slices $\left(\sim 1.5 \times 2 \times 3 \mathrm{~mm}^{3}\right)$, and it was determined to have eliminated almost completely the observable vessels in these data from the cluster set. No minimum cluster size was set because single-voxel "clusters" were not observed to contribute significantly to the final volume of interest.

From the total number of voxels that met the criterion that the cluster size be less than 35 voxels, we calculated the hypointensity burden (HIB), which we define as follows: HIB = (total number of voxels in accepted clusters/ total number of brain voxels) $\times$ (volume in $\mathrm{mm}^{3}$ of 1 voxel). The HIB gives the scaled percentage of brain volume that contains nonvessel hypointensities. The measure is useful because it allows us to detect both potential microbleeds as well as other more subtle forms of damage that could present as hypointensity, such as neural microstructural changes examined in the accompanying articles in this current series (Pasternak et al., ${ }^{41}$ and Sasaki et al. ${ }^{44}$ ). 
Advantages of this measure include the fact that it is completely automatic, the level of HIB can be investigated over time, and it can be compared at baseline to potentially characterize levels of previous damage. In addition, hypointensities that are not due to recent damage are not expected to change over time, and therefore add only a constant offset and would not affect the postconcussion HIB time course.

\section{Results}

Figure 1 shows orthogonal views from a representative subject; it depicts the SWI with an overlay of the discovered clusters. The exact shapes of the clusters are difficult to visualize in a single plane because clusters can be in more than a single slice. Note that obvious in-plane vessels are not discovered by the algorithm, nor are large vessels perpendicular to the plane of the image. Note also that, due to the small size of these clusters, they would not generally be classified as "traditional" CMBs (diameters larger than $\sim 5 \mathrm{~mm}$ ).

In Fig. 2, we show the HIB level of the 13 female subjects (Fig. 2 upper) and the 8 male subjects (Fig. 2 lower) in the nonconcussed group as a function of the number of self-reported previous concussions. There was no statistical difference in HIB level for the male subjects, regardless of the number of previous concussions. Note, however, that the HIB level was higher for the male than for the female subjects, even for the participants with no reported concussions, and this difference was statistically significant $(\mathrm{p}<$ $0.003,2$-tailed test, unequal variance) for that group. Note that there were not the same numbers of subjects at each number of previous concussions (see above in the discussion of the subjects for the multiplicity at each number). There was only a single female subject who had 1 previous
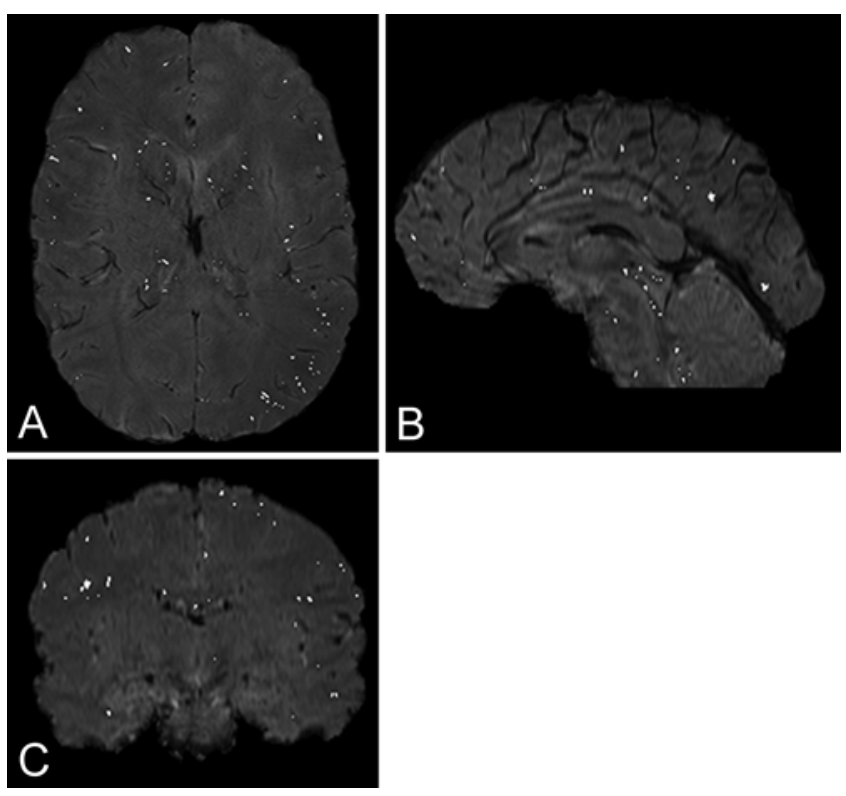

FIG. 1. A-C: Example of an SWI sequence with hypointensity clusters that fall below the intensity (intensity $<10 \%$ of maximum intensity) and maximal size (35 connected voxels) thresholds, and are shown as white and overlaid on the SWI. Note that obvious vessels or sulci are not selected by the algorithm.
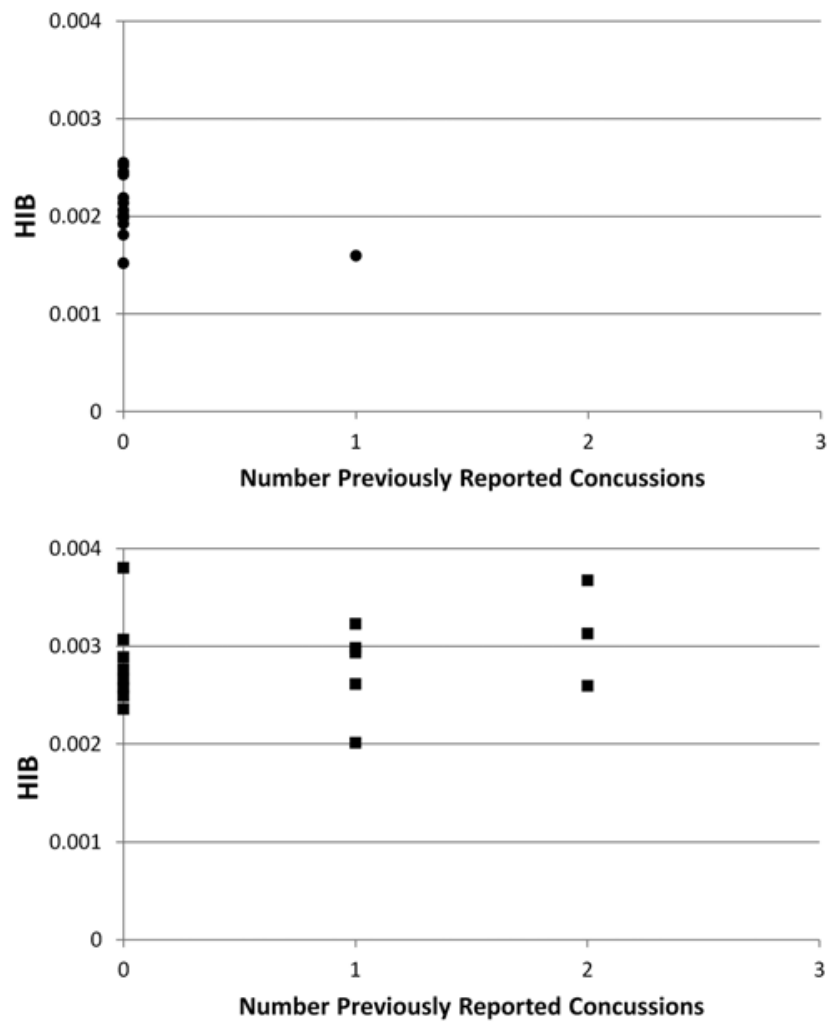

Fig. 2. Plots of the number of self-reported previous concussions for the nonconcussed group for the female subjects (upper) and the male subjects (lower). The difference in mean HIB values between subjects of each sex with 0 self-reported previous concussions in the nonconcussed group was statistically significant ( $p<0.003$, 2-tailed test, unequal variance).

concussion, and who also did not have an additional concussion during the season (nonconcussed group).

Figure 3 shows the HIB levels for all nonconcussed subjects (female subjects designated by circles and male subjects by squares) irrespective of previous concussions at each of the 2 imaging time points. Between sexes, the BOS and EOS data were significantly different from each other (BOS, $\mathrm{p}<0.0005$, EOS, $\mathrm{p}<0.05$; unpaired t-test, 2 -sided, unequal variance). The BOS and EOS data for the nonconcussed group did not, however, differ significantly from the BOS to the EOS within each sex.

In Fig. 4 we show the HIB versus time point curves for the concussion groups. Figure 4A shows the individual data for the 6 female subjects and Fig. 4B shows the individual data for the 5 male subjects. Note that for the male subjects, the last time point only includes data from 2 individuals; the others in this group did not complete the final imaging time point. The cohorts for both sexes consisted of participants who had HIB levels that changed over time and those for whom the HIB level remained constant ( 2 of 5 men, 2 of 6 women). The 1 female subject who completed only the BOS imaging time point, but later suffered a concussion, was not included in these data. For 2 of the subjects (1 male and 1 female), 1 of the postconcussion time points was coincident with the EOS time point for the other subjects. The data for these subjects were included in the calculations for, and 


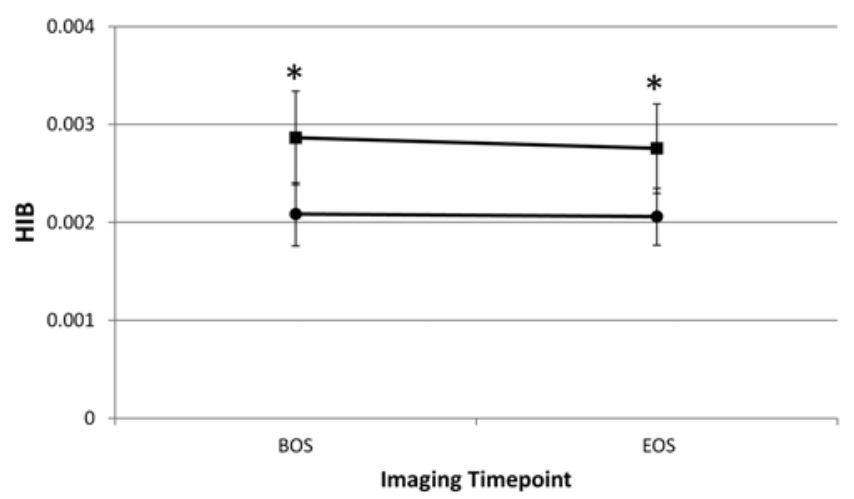

FIG. 3. Plot of the HIB levels for all nonconcussed subjects for females (circles) and males (squares) regardless of previous concussions at each of the 2 imaging time points. Asterisks indicate that between sexes, both the BOS and EOS time point data were significantly different from each other. Within each sex, however, the nonconcussed BOS and EOS data were not significantly different from each other, demonstrating that the HIB index is stable over the course of a season among the nonconcussed group's male and female subjects.

displayed with, the appropriate postconcussion time point rather than at EOS.

In Fig. 4C we show the mean \pm SD of the HIB for both sexes on a single plot to compare the HIB time course for each sex. The difference in mean HIB values between the sexes was statistically significant for the 2-week time point. Note that the overall change from BOS to HIB maximum was greater for the male than for the female subjects. We measured this increase by calculating the mean change in HIB at 2 weeks postconcussion compared to the BOS: $\Delta \mathrm{HIB}=\Sigma(\mathrm{HIB}(2$ weeks $)-\mathrm{HIB}(\mathrm{BOS})) / \mathrm{N}$, where $\mathrm{N}$ is the number of subjects, and found that $\triangle \mathrm{HIB}$ (female) $=$ $0.0003 \pm 0.0004$, whereas $\Delta$ HIB (male) $=0.0006 \pm 0.0007$. Note that although the mean HIB level had its peak at the 72-hour time point in women in this study, this may be due to the fact that SWI could be obtained in only half of the female subjects at this time point. It should also be observed that there were subjects for each sex whose HIB value generally did not change with time.

Figure 5 displays the mean $\pm \mathrm{SD}$ for the concussion group and all nonconcussed subjects, regardless of previous concussion status, for female subjects (Fig. 5 upper) and male subjects (Fig. 5 lower). The concussed subjects are represented by the diamonds and the nonconcussed subjects by the triangles. Note that the BOS and EOS data were almost identical for female subjects in both cohorts, but that this was not the case for male subjects. In male subjects, the EOS and BOS levels in the nonconcussed group were higher than those for the concussed cohort.

The HIB data at each time point were tested for statistical significance, versus that at the BOS time point for both sexes. Only the 2-week time point for the concussed male subjects' data showed a statistically significant increase over the BOS data (asterisk in Fig. 5 lower).

\section{Discussion}

\section{Study Overview}

Although most studies use visual inspection to detect

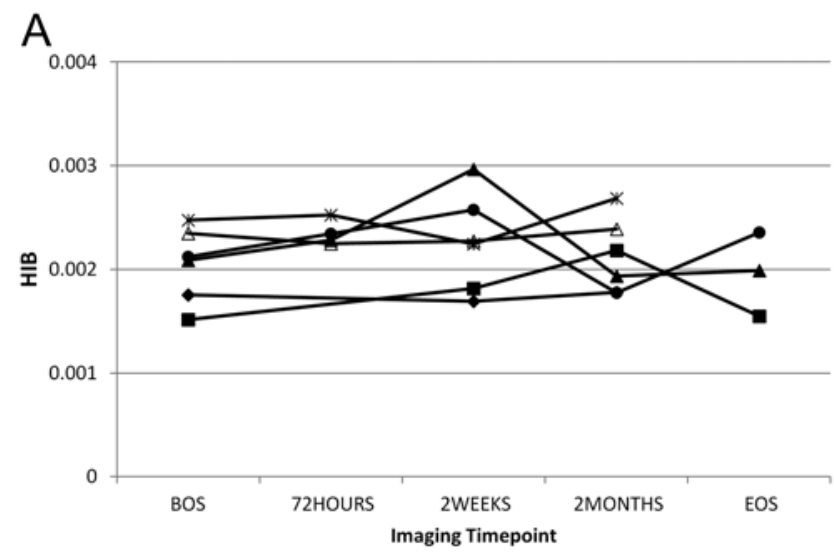

B

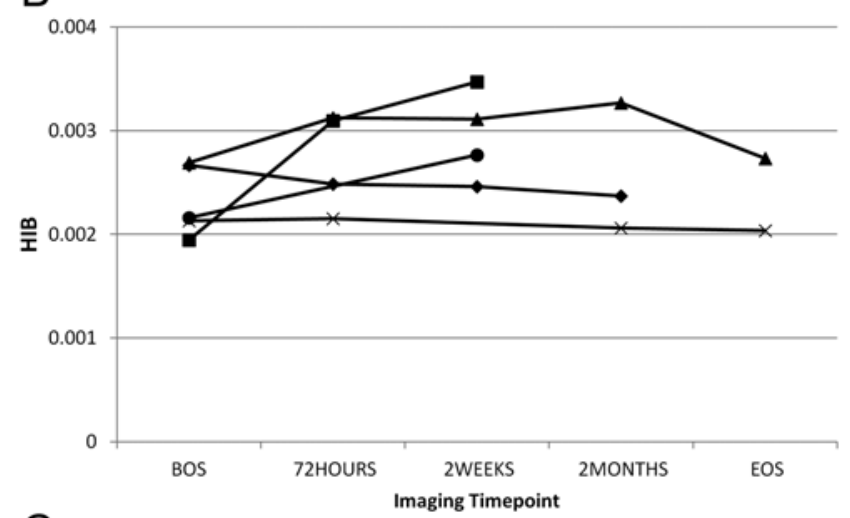

C

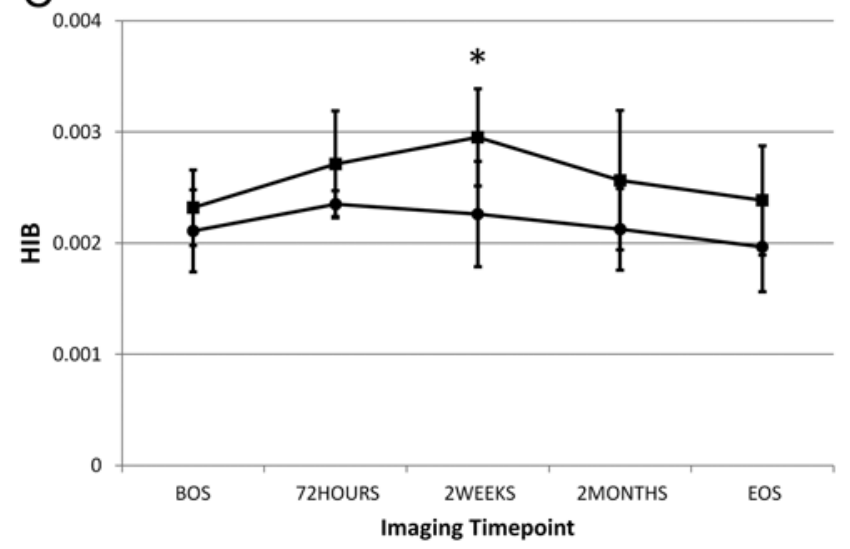

FIG. 4. Plots of the HIB versus time point curves for individual subjects for the concussed groups for each sex (A, 6 female subjects and B, 5 male subjects). For 2 of the subjects ( 1 man and 1 woman), 1 of the postconcussion time points was coincident with the EOS time point for the other subjects. The data for these subjects were included in the calculations for, and displayed with, the appropriate postconcussion time point rather than at the EOS. In addition we show the mean HIB for both sexes (C) to compare the HIB time courses for each sex. Note that the overall change from the BOS to the mean HIB maximum is greater for the male than for the female subjects. Asterisk in panel $C$ denotes a statistically significant difference, with squares representing data for male subjects and circles data for female subjects.

CMBs, this strategy is less useful for cases of milder injury that may not produce lesions with enough reduced image intensity (or volume) to be detected by the eye alone. One must also be careful not to interpret the discovered 

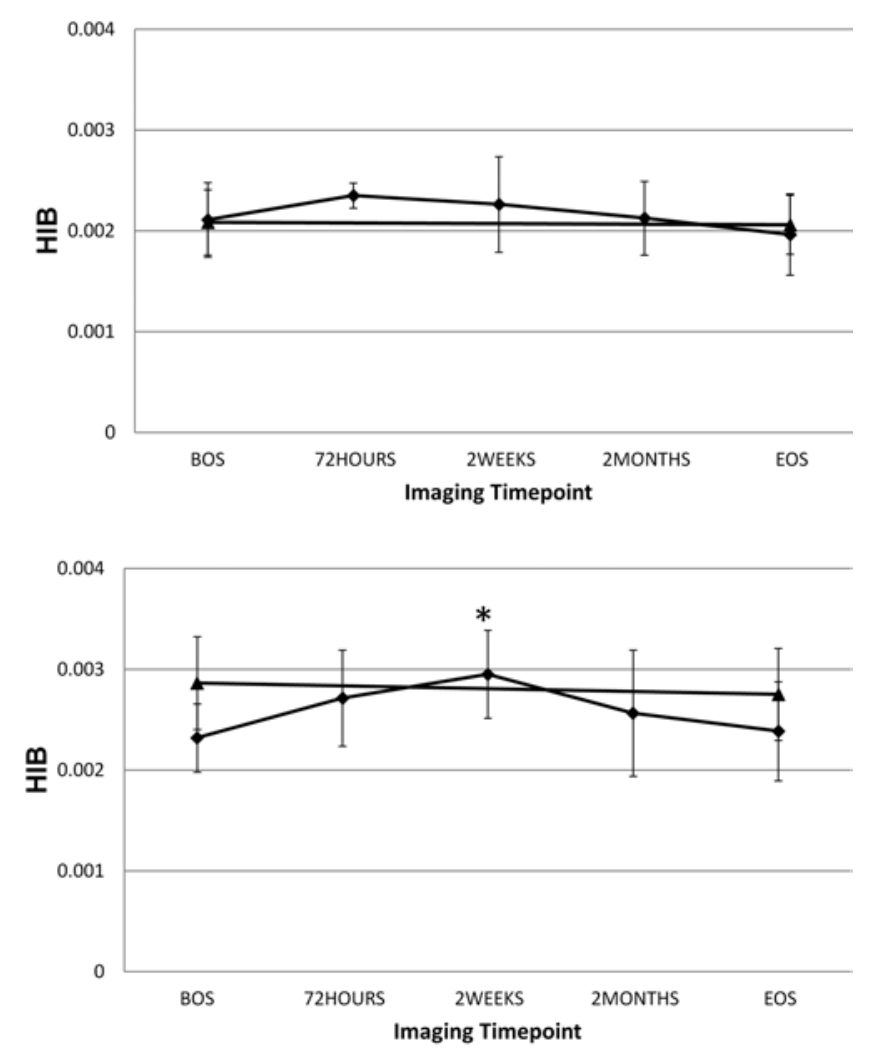

FIG. 5. Graphs showing the mean \pm SD HIB for the concussed (diamonds) and all nonconcussed (triangles) subjects regardless of previous concussion status for the female subjects (upper) and the male subjects (lower). The BOS and EOS data are almost identical for nonconcussed and concussed cohorts for the female subjects, but the mean BOS and EOS HIB values for the nonconcussed group are higher, except at 2 weeks, than either of the concussed time points for the male subjects. The asterisk in the lower panel denotes a statistically significant difference between the 2-week time point data for the concussed male subjects and their BOS mean HIB value.

areas depicted in Fig. 1 as traditionally defined CMBs (for example, $>5.7 \mathrm{~mm}$ in patients with cerebral amyloid angiopathy $\left.{ }^{30}\right)$. Here we tailor our analysis method to the expectation that there will be few if any CMBs of that size in our cohort and that the damage may be occurring at a much smaller-length scale. We note that there have been recent observations ${ }^{13,19}$ of submillimeter pericapillary hemosiderin, which may be contributing to the clusters discovered in this work.

We also note that we make no claim that this simple method specifically detects microbleeds, and there will inevitably be a background of false-positive detections. For example, there are a few small clusters present in the aqueduct in Fig. 1. However, we believe that, given the time course of the HIB metric postconcussion, we are also detecting changes that are specific to the concussion. Although it is true that there will be a baseline of clusters detected that are not due to the concussion, this baseline will be manifest as a common offset for each group. This common offset does not, however, explain the sex-based difference in the BOS HIB, which will be discussed below.

Given that each voxel may produce a signal that arises from tissue in multiple states of health or injury, the voxel intensity may not be reduced to the same extent as it is in larger or multiple lesions resulting from stroke or more severe trauma. Although we observed few visually obvious $(>5 \mathrm{~mm})$ signs of damage in these data, we did find, by characterizing and following clusters of the lowest-intensity voxels, time-dependent behavior of our HIB metric after concussion. In addition we note that, in the nonconcussed group data, the BOS and EOS HIB levels were the same, implying that the observed time dependence of HIB in the concussed group data was not due to the hardware or threshold, but rather was related to the concussion. It is also important to note that we are only discovering a population of connected voxels of a certain size. This was done to reduce the possibility of detecting vessels with the algorithm, but note that large CMBs would also not be detected, and therefore the algorithm is tuned to our current application. This method is also fully automatic and less computationally intensive than the method of Barnes et al., ${ }^{4}$ which uses support vector machines to separate CMBs from other hypointensities.

\section{Hardware Upgrade}

One complication in the analysis of these data was that the gradient coil was changed during the season. However, it is not likely that this change would affect the selection of the lowest $10 \%$ of image intensities because it is unlikely that the gradients would decrease higher image intensities into the lowest decile in significant numbers sufficient to affect the results presented here. We also found no evidence of regional heterogeneity in the final clusters selected for analysis. In addition, we found no statistically significant difference in image intensity range, median, or maximum value from the BOS to EOS time points (within the sexes). In addition, because the within-group HIB in all groups is statistically the same for BOS and EOS, this strongly suggests that the coil change was not a significant factor in the results of this analysis.

\section{Sex-Based Differences in the HIB Metric}

To our knowledge, this is the first study to examine sex-based differences in patients with sports-related concussions that included prospective incidence rates and evidence of organic brain injury. The first striking feature of Figs. 2 and 3 is the difference in HIB level between the male and female subjects. This difference occurred at all time points, and for both the nonconsussed and concussed groups. One possible explanation for the difference in the BOS HIB level between the sexes is that the HIB lowest $10 \%$ threshold is different between the sexes, and therefore the difference in threshold allows more clusters to be discovered. There was, in fact, a statistically significant difference in the HIB lowest $10 \%$ threshold between the male and female subjects in the nonconcussed group data (using that as the data set with the largest number of individuals). This was true for both the BOS ( $p<$ 0.002 , 2-tailed, unequal variance) and the EOS ( $p<0.05$, 2-tailed, unequal variance).

One would expect that the data with the largest threshold would have the largest range of values (assuming that 
the intensity-value histograms have similar shapes), but this was not the case. We find that although the female subjects had the largest intensity threshold value-mean and median - the ranges of intensity values were larger for the male subjects. Therefore, the SWI intensity-value distributions were statistically different between the sexes, but not in a way that explains the sex-based difference in HIB values; that is, for a higher threshold one would expect more clusters to be detected and therefore a higher detected HIB. In fact, the HIB level for the female subjects was less than that for the male subjects in both groups. In addition, there was no statistically significant difference in the threshold between time points within each sex.

One hypothesis for the shift of HIB to lower levels is that the male subjects may have accumulated more subconcussive hits resulting in hypointensities over their careers and that this resulted in cumulative damage and/or HIB and higher BOS HIB. In addition, whereas the total number of previous concussions was approximately the same for each sex in the concussed group, the distribution among subjects was not the same. More specifically, all of the male subjects in the concussed group had had 1 or more previous concussions, whereas this was not the case for the female subjects (see more detailed discussion below). It should be noted that although the number of previous concussions was recorded in this study, these were self-reported, and no other information regarding the concussions was available.

The time courses of mean HIB for the male and female subjects were roughly equivalent, but there is obviously a range of behavior in the individual subjects. In addition, the maximum increase in HIB was greater for the male subjects. This greater increase, although not statistically significant due to the small number of subjects in each group (and also taking into account that there was little change over time for 2 subjects in each group), was nonetheless suggestive of a larger effect for the male than for the female subjects. This may be due to differences in degree of trauma for these particular individuals, but should be investigated with a larger cohort. It is also interesting that the HIB level renormalized by the EOS time point for both sexes.

\section{Previous Concussions}

Figure 5 allows the direct comparison of the nonconcussed and concussed group data. Note that the BOS and EOS HIB levels were identical for both groups of female subjects, but not for the male subjects. This may be due to several factors. First, the number of previous concussions in the concussed female subjects was $\{4,3,0,0,0,1\}$, given in order of highest to lowest BOS HIB level, whereas for the concussed male subjects the number of previous concussions was $\{1,2,3,1,2\}$. It is interesting that the number of previous concussions correlated well with the observed BOS HIB level in the female subjects. The picture from the concussed group data of the male subjects is more complicated, and points to the importance of a method for estimating cerebral damage postconcussion.

As noted earlier in this paper, the number of previous concussions was self-reported, and there is a tendency for the male subjects to underreport the number and sever- ity of concussions. ${ }^{36}$ In addition, the nonconcussed cohort of male subjects reported more previous concussions (11 total in 16 males) than did the female cohort (1 total in 14 females), and this could account for the higher BOS and EOS HIB levels in the nonconcussed group of male subjects. Assuming that the HIB level reflects damage due to concussion, the smaller number of previous concussions in the female subjects is consistent with the lower overall HIB level in the nonconcussed group, and with the BOS and EOS levels matching at those time points for both groups of female subjects. In addition, the sole statistically significant increase in postconcussion HIB versus BOS HIB was observed in the cohort of male subjects. This, along with the fact that each individual in the concussed group of male subjects had previous concussions, is consistent with the idea that damage due to concussions may be cumulative. ${ }^{27}$ Hockey players are a unique group, both because of the observed rate of concussion in the sport for both sexes, and because the nature of routine play is such that it is unlikely that a player can avoid any sort of trauma to the head (even when concussions are neither identified nor self-reported). Given what is known about the occurrence of multiple subconcussive blows and later concussions, this method may be useful in the detection of cumulative effects of concussion.

\section{Participation Rates}

We also note that there was a lower participation rate among the male subjects than the female subjects-the male subjects failed to undergo imaging at the 2-month and EOS time points at a higher rate than the female subjects. The documented participation rate demonstrates the difficulty inherent in these studies. The participation goals of the athlete and the researcher conflict, because the identification of concussion results in the injured athlete being restricted from competition.

\section{Study Limitations}

There are several limitations in this study. The strength of this study was that independent specialist medical observers were involved in direct concussion identification and diagnosis. Therefore it was more likely that a concussion would be identified and recorded. This independent direct observation and diagnosis maximizes the number of concussions that would be identified for each group. Subjects were also broken into groups differentiated by sex and diagnosis (concussion/no concussion). However, not all subjects who suffered a concussion during the season completed all of the scheduled imaging time points. This was primarily due to player perception that participation in the study had the potential to curtail his or her ability to return to play after a concussion. Interestingly, overall participation was greater among the female than among the male subjects. Given the variability in subject outcome and response to concussion, the low numbers in each group mean that large effects in HIB are needed to achieve statistical significance.

Another limitation of this study was that the Immediate Postconcussion Assessment and Cognitive Test (ImPACT) and Sport Concussion Assessment Tool-2 


\section{Susceptibility-weighted imaging study in ice hockey players}

(SCAT2) scores collected were also highly variable. Echlin and colleagues, ${ }^{16}$ in an earlier publication that presented the clinical outcomes data, attributed this to one or more of the following: the small sample size, the inherent variability of the tests, or the possibility of malingering of subjects in the baseline tests. Therefore, it is not surprising that no correlation was found between the HIB metric and the ImPACT or SCAT2 scores (data not shown). However, in that earlier study we did find that a statistically significant effect was present for the SCAT2 total symptom score for concussed subjects, which increased (the SCAT2 total symptom score increases as the number of self-perceived symptoms and severity of symptoms increase) at postconcussion and renormalized by the end of the season, similar to the time course of the HIB. Nonetheless, given the factors discussed above, further study of this metric in other populations, and in comparison with pathological data, will be needed to make further inferences regarding a link between the HIB and clinical outcomes.

Another limitation is that imaging was not performed at one or more points during the season for subjects not suffering a concussion. These data would have aided in characterizing the reliability of the HIB metric and would have helped to put the magnitude of changes observed in the concussed subjects in context.

\section{Conclusions}

The SWI modality allows us to detect hypointense brain regions that have been shown to be associated with the presence of blood-breakdown products originating from damaged vessels. This method extends the utility of SWI from the enhancement and detection of larger (>5 mm) CMBs that are often observed in more severe TBI to concussion in which visual detection of injury is difficult. The HIB metric proposed here shows statistically significant changes over time in the male subjects. A smaller nonsignificant increase in the burden metric was observed in the female subjects. Data acquired using this method could be used for the monitoring of players throughout their careers and could lead to improved diagnoses and return-to-play guidelines.

\section{Disclosure}

The authors report no conflict of interest concerning the materials or methods used in this study or the findings specified in this paper. Funding for this work was provided to the HCEP (Hockey Concussion Education Project) and Dr. Echlin by the Ontario Trillium Foundation, the Dave Irwin Foundation for Brain Injury, the Ontario Neurotrauma Foundation, Air Canada, The Ontario Ministry of Health and Long-Term Care, The Ontario Ministry of Tourism, Culture and Sport, and The Ontario Ministry of Education. This work was partially funded by grants from the NIH (P41RR013218, P41EB015902, R01MH074794), the Department of Defense (X81XWH-07-CC-CSDoD), and a VA merit grant. Dr. Pasternak was partially supported by a NARSAD (National Alliance for Research on Schizophrenia and Depression) Young Investigator grant from the Brain \& Behavior Research Foundation. Dr. Koerte is supported by the Else Kröner-Fresenius Foundation, Germany. Dr. Sasaki is supported by the Strategic Young Researcher Overseas Visits Program for Accelerating Brain Circulation from the Japan Society for the Promotion of Science. Mr. Mayinger is supported by the Petraeic Legate Foundation. Dr. Shenton is a consultant on an NIH grant to the State University of New York on velocardiofascial syndrome, and on a grant supported by the Henry Jackson Foundation.

Author contributions to the study and manuscript preparation include the following. Conception and design: Echlin, Helmer, Pasternak, Preciado, Koerte, Forwell, Shenton. Acquisition of data: Echlin, Forwell, Skopelja. Analysis and interpretation of data: Echlin, Helmer, Pasternak, Fredman, Preciado, Sasaki, Mayinger, Johnson, Holmes, Shenton. Drafting the article: Echlin, Helmer, Pasternak, Fredman, Koerte, Sasaki, Mayinger, Holmes, Skopelja, Shenton. Critically revising the article: all authors. Reviewed submitted version of manuscript: all authors. Approved the final version of the manuscript on behalf of all authors: Echlin. Statistical analysis: Helmer, Preciado, Johnson, Shenton. Administrative/technical/ material support: Echlin, Helmer, Preciado, Forwell, Skopelja, Shenton. Study supervision: Echlin, Helmer, Koerte, Forwell, Shenton. Librarian support: Skopelja.

\section{References}

1. Agel J, Dick R, Nelson B, Marshall SW, Dompier TP: Descriptive epidemiology of collegiate women's ice hockey injuries: National Collegiate Athletic Association Injury Surveillance System, 2000-2001 through 2003-2004. J Athl Train 42:249-254, 2007

2. Agel J, Dompier TP, Dick R, Marshall SW: Descriptive epidemiology of collegiate men's ice hockey injuries: National Collegiate Athletic Association Injury Surveillance System, 19881989 through 2003-2004. J Athl Train 42:241-248, 2007

3. Ashikaga R, Araki Y, Ishida O: MRI of head injury using FLAIR. Neuroradiology 39:239-242, 1997

4. Barnes SR, Haacke EM, Ayaz M, Boikov AS, Kirsch W, Kido D: Semiautomated detection of cerebral microbleeds in magnetic resonance images. Magn Reson Imaging 29:844-852, 2011

5. Barr WB: Neuropsychological testing of high school athletes. Preliminary norms and test-retest indices. Arch Clin Neuropsychol 18:91-101, 2003

6. Borg J, Holm L, Cassidy JD, Peloso PM, Carroll LJ, von Holst $\mathrm{H}$, et al: Diagnostic procedures in mild traumatic brain injury: results of the WHO Collaborating Centre Task Force on Mild Traumatic Brain Injury. J Rehabil Med 36 (43 Suppl):61-75, 2004

7. Chan S, Kartha K, Yoon SS, Desmond DW, Hilal SK: Multifocal hypointense cerebral lesions on gradient-echo MR are associated with chronic hypertension. AJNR Am J Neuroradiol 17:1821-1827, 1996

8. Charidimou A, Jäger HR, Werring DJ: Cerebral microbleed detection and mapping: principles, methodological aspects and rationale in vascular dementia. Exp Gerontol 47:843-852, 2012

9. Covassin T, Swanik CB, Sachs M, Kendrick Z, Schatz P, Zillmer E, et al: Sex differences in baseline neuropsychological function and concussion symptoms of collegiate athletes. Br J Sports Med 40:923-927, 2006

10. Dale AM, Fischl B, Sereno MI: Cortical surface-based analysis. I. Segmentation and surface reconstruction. Neuroimage 9:179-194, 1999

11. Daneshvar DH, Nowinski CJ, McKee AC, Cantu RC: The epidemiology of sport-related concussion. Clin Sports Med 30: 1-17, vii, 2011

12. Dashnaw ML, Petraglia AL, Bailes JE: An overview of the basic science of concussion and subconcussion: where we are and where we are going. Neurosurg Focus 33(6):E5, 2012

13. De Reuck J, Deramecourt V, Cordonnier C, Leys D, Maurage CA, Pasquier F: The impact of cerebral amyloid angiopathy on the occurrence of cerebrovascular lesions in demented patients with Alzheimer features: a neuropathological study. Eur J Neurol 18:913-918, 2011 
14. Dick RW: Is there a gender difference in concussion incidence and outcomes? Br J Sports Med 43 (Suppl 1):i46-i50, 2009

15. Doezema D, King JN, Tandberg D, Espinosa MC, Orrison WW: Magnetic resonance imaging in minor head injury. Ann Emerg Med 20:1281-1285, 1991

16. Echlin PS, Skopelja EN, Worsley R, Dadachanji SB, LloydSmith DR, Taunton JA, et al: A prospective study of physicianobserved concussion during a varsity university ice hockey season: incidence and neuropsychological changes. Part 2 of 4. Neurosurg Focus 33(6):E2, 2012

17. Farace E, Alves WM: Do women fare worse: a metaanalysis of gender differences in traumatic brain injury outcome. J Neurosurg 93:539-545, 2000

18. Faul MD, Xu L, Wald MM, Coronado VG: Traumatic Brain Injury in the United States: Emergency Department Visits, Hospitalizations, and Deaths, 2002-2006. Atlanta: Centers for Disease Control, 2010

19. Fisher M, French S, Ji P, Kim RC: Cerebral microbleeds in the elderly: a pathological analysis. Stroke 41:2782-2785, 2010

20. Gentry LR, Godersky JC, Thompson B: MR imaging of head trauma: review of the distribution and radiopathologic features of traumatic lesions. AJR Am J Roentgenol 150:663672,1988

21. Gentry LR, Godersky JC, Thompson B, Dunn VD: Prospective comparative study of intermediate-field MR and CT in the evaluation of closed head trauma. AJR Am J Roentgenol 150:673-682, 1988

22. Haacke EM, Mittal S, Wu Z, Neelavalli J, Cheng YC: Susceptibility-weighted imaging: technical aspects and clinical applications, part 1. AJNR Am J Neuroradiol 30:19-30, 2009

23. Haacke EM, Xu Y, Cheng YC, Reichenbach JR: Susceptibility weighted imaging (SWI). Magn Reson Med 52:612-618, 2004

24. Haddar D, Haacke E, Sehgal V, Delproposto Z, Salamon G, Seror O, et al: [Susceptibility weighted imaging. Theory and applications.] J Radiol 85:1901-1908, 2004 (Fr)

25. Hofman PA, Stapert SZ, van Kroonenburgh MJ, Jolles J, de Kruijk J, Wilmink JT: MR imaging, single-photon emission $\mathrm{CT}$, and neurocognitive performance after mild traumatic brain injury. AJNR Am J Neuroradiol 22:441-449, 2001

26. Hughes DG, Jackson A, Mason DL, Berry E, Hollis S, Yates DW: Abnormalities on magnetic resonance imaging seen acutely following mild traumatic brain injury: correlation with neuropsychological tests and delayed recovery. Neuroradiology 46:550-558, 2004

27. Iverson GL, Gaetz M, Lovell MR, Collins MW: Cumulative effects of concussion in amateur athletes. Brain Inj 18:433443, 2004

28. Jeerakathil T, Wolf PA, Beiser A, Hald JK, Au R, Kase CS, et al: Cerebral microbleeds: prevalence and associations with cardiovascular risk factors in the Framingham Study. Stroke 35:1831-1835, 2004

29. Jenkinson M, Beckmann CF, Behrens TE, Woolrich MW, Smith SM: FSL. Neuroimage 62:782-790, 2012

30. Kidwell CS, Greenberg SM: Red meets white: do microbleeds link hemorrhagic and ischemic cerebrovascular disease? Neurology 73:1614-1615, 2009

31. Koerte IK, Kaufmann D, Hartl E, Bouix S, Pasternak O, Kubicki M, et al: A prospective study of physician-observed concussion during a varsity university hockey season: white matter integrity in ice hockey players. Part 3 of 4 . Neurosurg Focus 33(6):E3, 2012

32. Le TH, Gean AD: Neuroimaging of traumatic brain injury. Mt Sinai J Med 76:145-162, 2009

33. Lewine JD, Davis JT, Bigler ED, Thoma R, Hill D, Funke M, et al: Objective documentation of traumatic brain injury sub- sequent to mild head trauma: multimodal brain imaging with MEG, SPECT, and MRI. J Head Trauma Rehabil 22:141155,2007

34. Mansell JL, Tierney RT, Higgins M, McDevitt J, Toone N, Glutting J: Concussive signs and symptoms following head impacts in collegiate athletes. Brain Inj 24:1070-1074, 2010

35. Maruta J, Lee SW, Jacobs EF, Ghajar J: A unified science of concussion. Ann N Y Acad Sci 1208:58-66, 2010

36. McCrea M, Hammeke T, Olsen G, Leo P, Guskiewicz K: Unreported concussion in high school football players: implications for prevention. Clin J Sport Med 14:13-17, 2004

37. Meythaler JM, Peduzzi JD, Eleftheriou E, Novack TA: Current concepts: diffuse axonal injury-associated traumatic brain injury. Arch Phys Med Rehabil 82:1461-1471, 2001

38. Mittal S, Wu Z, Neelavalli J, Haacke EM: Susceptibilityweighted imaging: technical aspects and clinical applications, part 2. AJNR Am J Neuroradiol 30:232-252, 2009

39. Nandigam RN, Viswanathan A, Delgado P, Skehan ME, Smith EE, Rosand J, et al: MR imaging detection of cerebral microbleeds: effect of susceptibility-weighted imaging, section thickness, and field strength. AJNR Am J Neuroradiol 30:338-343, 2009

40. Oppenheimer DR: Microscopic lesions in the brain following head injury. J Neurol Neurosurg Psychiatry 31:299-306, 1968

41. Pasternak O, Koerte IK, Bouix S, Fredman E, Sasaki T, Mayinger M, et al: Hockey Concussion Education Project, Part 2. Microstructural white matter alterations in acutely concussed ice hockey players: a longitudinal free-water MRI study. Clinical article. J Neurosurg [epub ahead of print February 4, 2014. DOI: 10.3171/2013.12.JNS132090]

42. Pellman EJ, Viano DC, Tucker AM, Casson IR: Concussion in professional football: location and direction of helmet impacts-Part 2. Neurosurgery 53:1328-1341, 2003

43. Pellman EJ, Viano DC, Tucker AM, Casson IR, Waeckerle JF: Concussion in professional football: reconstruction of game impacts and injuries. Neurosurgery 53:799-814, 2003

44. Sasaki T, Pasternak O, Mayinger M, Muehlmann M, Savadjiev P, Bouix S, et al: Hockey Concussion Education Project, Part 3. White matter microstructure in ice hockey players with a history of concussion: a diffusion tensor imaging study. Clinical article. J Neurosurg [epub ahead of print February 4, 2014. DOI: 10.3171/2013.12.JNS132092]

45. Schick DM, Meeuwisse WH: Injury rates and profiles in female ice hockey players. Am J Sports Med 31:47-52, 2003

46. Smith DH, Meaney DF, Shull WH: Diffuse axonal injury in head trauma. J Head Trauma Rehabil 18:307-316, 2003

47. Smith SM: Fast robust automated brain extraction. Hum Brain Mapp 17:143-155, 2002

48. Spain A, Daumas S, Lifshitz J, Rhodes J, Andrews PJ, Horsburgh K, et al: Mild fluid percussion injury in mice produces evolving selective axonal pathology and cognitive deficits relevant to human brain injury. J Neurotrauma 27:1429-1438, 2010

49. Voller B, Benke T, Benedetto K, Schnider P, Auff E, Aichner F: Neuropsychological, MRI and EEG findings after very mild traumatic brain injury. Brain Inj 13:821-827, 1999

Manuscript submitted September 2, 2013.

Accepted December 3, 2013.

Please include this information when citing this paper: published online February 4, 2014; DOI: 10.3171/2013.12.JNS132093.

Address correspondence to: Paul S. Echlin, M.D., Elliott Sports Medicine Clinic, 1100 Walkers Line, Ste. 2, Burlington, ON, Canada L7N 2G3. email: psechlin@gmail.com. 